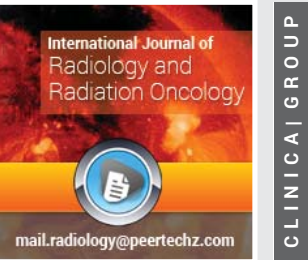

\section{Dukho $\mathrm{AJ}^{1 *}$ and Nazima Dharsee ${ }^{2}$}

'Department of Radiation Therapy, Muhimbili University of Health and Allied Sciences (MUHAS), P.O.Box 65001, Dar es Salaam, Tanzania 2Department of Clinical Oncology, Ocean Road Cancer Institute (ORCI), P.O Box 3592 Dar es Salaam, Tanzania

Received: 03 August, 2018

Accepted: 22 August, 2018

Published: 23 August, 2018

*Corresponding authors: Dukho AJ, Department of Radiation Therapy, Muhimbili University of Health and Allied Sciences (MUHAS), P.O.Box 65001, Dar es Salaam, Tanzania, E-mail: dukhoandrew@gmail.com

Nazima Dharsee, Department of Clinical Oncology, Ocean Road Cancer Institute (ORCI), P.O Box 3592 Dar es Salaam, Tanzania, E-mail: njdharsee@gmail.com

Keywords: Head and neck cancer; Comparative evaluation; Immobilization devices; Thermoplastic mask; LS -2000; ORCI

https://www.peertechz.com
Research Article

A comparative evaluation of two head and neck immobilization devices using verification film for head and neck cancer patients receiving radiation therapy at Ocean Road Cancer Institute (ORCI) aero-digestive tract

\section{Abstract}

Background: Accurate and reproducible patient positioning is fundamental to the success of fractionated radiotherapy. To achieve accurate daily treatment delivery, various immobilization devices are used in radiotherapy departments. At $\mathrm{ORCl}$ the mostly used immobilization devices for radiotherapy treatment of head and neck cancer patients are thermoplastic mask and LS-2000. However, the effectiveness and accuracy of these immobilization devices in daily set-up is not known. This study was performed to evaluate accuracy and reproducibility of patient setup for both thermoplastic mask and LS2000 devices, during irradiation of head and neck tumors.

Methods: 30 head and neck cancer patients treated at ORCI between May 2017 and July 2017 were enrolled. 16 patients were immobilized with LS-2000, while 14 patients were immobilized with thermoplastic mask. All patients attended planning session which was done using Tera-Six simulator, and treatment was done using a CO-60 machine. Portal films were taken on the initial treatment day and once per week for three weeks of treatment. 90 portal images were analyzed to assess patient displacement in antero-posterior and supero-inferior directions by measuring distance from center of treatment field to clearly visible bony landmark using a ruler.

Results: The ranges of set up deviation for both thermoplastic mask and LS-2000 immobilization devices were $(-0.53 \mathrm{~cm}$ to $+0.67 \mathrm{~cm})$ and $(-0.8$ to $+1.42 \mathrm{~cm})$ in AP direction, and $(-0.53 \mathrm{~cm}$ to $+1.07 \mathrm{~cm})$ and $(-0.47 \mathrm{~cm}$ to $2.23 \mathrm{~cm})$ in SI direction. Seventy eight point six percent $(78.6 \%)$ and seventy five percent ( $75 \%$ ) of setup deviations were within $\pm 0.4 \mathrm{~cm}$ in AP direction for both thermoplastic mask and LS-2000 immobilization devices respectively. In SI direction fifty seven (57.1\%) and fifty percent (50\%) of setup deviation were within $\pm 0.4 \mathrm{~cm}$, for both thermoplastic mask and LS-2000 devices respectively. The random errors for the thermoplastic mask and LS-2000 devices were $\pm 0.34 \mathrm{~cm}$ and $\pm 0.62 \mathrm{~cm}$ in AP direction and \pm $0.48 \mathrm{~cm}$ and $\pm 0.64 \mathrm{~cm}$ in SI direction respectively. The systematic deviations were $\pm 0.35 \mathrm{~cm}$ and $\pm 0.62 \mathrm{~cm}$ in AP direction and $\pm 0.5 \mathrm{~cm}$ and $\pm 0.64 \mathrm{~cm}$ in SI direction for the thermoplastic mask and LS-2000 devices respectively.

Conclusion: Thermoplastic mask for immobilization of HNC treatment provides a field placement reproducibility that was better than LS-2000 as it demonstrated smaller translational set up displacements in both AP and SI directions.

\section{Abbreviations}

AP: Antero -posterior; Cm: centimeter; CO-60: Cobalt 60; C-C: Cranio-caudal; HNC: Head and Neck Cancer; IMRT: Intensity Modulated Radiotherapy; ML: Medio-Lateral; NPC: Nasopharyngeal Carcinoma; ORCI: Ocean Road Cancer Institute; PBRT: Proton Beam Radiotherapy; SI: Supero-inferior; RT:
Radiotherapy; 2DRT: Two dimensional Radiotherapy; 3DCRT: Three dimensional Radiotherapy

\section{Introduction}

Head and neck cancer comprises a heterogeneous group of primary tumors involving upper aero-digestive tract, paranasal 
sinuses, salivary and thyroid glands [1]. They rank six among the most common cancers worldwide, and it is estimated that there are more than 640,000 new cases of HNC and 350,000 deaths occurring each year worldwide [2]. Due to paucity of published data regarding head and neck cancers in Tanzania, there is difficult to appreciate the pattern and magnitude of the problem in the country [3].

The optimal management of head and neck cancer requires a multidisciplinary approach, whichinclude surgery, radiotherapy and chemotherapy [4]. Radiotherapy is the main stay and it may be used alone or as a part of multimodality approach. The main challenge in radiotherapy for any type of cancer is to attain the highest probability of tumor control or cure with the least amount of morbidity and toxicity to normal surrounding tissues and critical organs [1]. Radiotherapy for patients with head and neck cancer is extremely complexdue to the close proximity of critical organs [5].

Radiation therapy designs have evolved over the past 30 years from being based on two- dimensional (2D) to threedimensional (3D) images, incorporating increasingly complex computer algorithms such as 3DCRT, IMRT, IGRT and proton beam therapy (PBRT). 2DRT consists of a single beam from one to four directions with the radiation fields designed on $2 \mathrm{D}$ fluoroscopic simulation images. Beam setups are usually quite simple; plans frequently consist of opposed lateral fields or four fields [6].

In $2 \mathrm{D}$ radiotherapy, a patient has one planning session followed by many treatment sessions. In the planning phase, the patient's simulator image is used to establish a treatment plan. The aim is to deliver this plan in all treatment sessions.

At Ocean Road Cancer Institute, (ORCI), head and neck cancer patients are treated using $2 \mathrm{D}$ radiotherapy planning and delivery. This treatment technique is used in resource limited settings due to its affordability but is more liable to errors compared to advanced technologies such as IGRT where real time images can be obtained during treatment and verification done during each treatment session. Thus, in 2D radiotherapy, it is important to ensure proper patient immobilization owing to the fact that daily treatment verification is not feasible.

Immobilization devices have two fundamental roles: to immobilize the patient during treatment and to provide a reliable means of reproducing the patient position from simulation to treatment, and from one treatment to another. Moreover, a well-constructed immobilization system may reduce the daily positioning time of the patient and make the patient feel more secure and less anxious.

In HNC radiation therapy, variations in practice exist between different hospitals, ranging from the use of a simple device such as masking tape, large rubber bands or a bite block and LS-2000, to devices made of plaster, plastic and Styrofoam.

In our center, we traditionally use a simple immobilization device (LS-2000) and thermoplastic mask for positioning of head and neck cancer patients during treatment planning and delivery of radiation therapy. However, the effectiveness and accuracy of immobilization devices in daily set-up is not clearly known. This study aims to evaluate the accuracy and reproducibility of thermoplastic mask and LS-2000 immobilization devices.

\section{Materials and Methods}

This was a hospital based prospective, study to compare setup errors between two immobilization devices namely Thermoplastic mask and LS-2000 frame. Thirty head and neck cancer patients treated at ORCI between May 2017 and July 2017 were conveniently enrolled. These patients were randomly assigned to two groups representingtwo immobilization devices used in the treatment set up; 14 patients with a thermoplastic mask, and 16 patients with an LS-2000. All patients attended planning session which was done using Tera-Six simulator where field sizes, gantry angles, collimator angles and references of the treatment field to anatomical landmarks of the head and neck cancer patients were determined for appropriate beam placement. The treatment beam arrangement consisted of two parallel opposed lateral fields covering the head and neck target and direct anterior field for some patients with positive supraclavicular nodes. All patients were treated on a Co-60, Theratron Equinox $100 \mathrm{~cm}$ SAD treatment machine Portal images were obtained weekly using verification films.

Thermoplastic mask and LS-2000 with their accessories were evaluated in this study. To form thermoplastic mask in the mould room, thermoplastic material was dipped into a hot water bath of about 75 degree Celsius. The patient was positioned supine on a headrest best fitting their head and neck, and that was attached to a carbon fiber base plate. After being heated thermoplastic material becomes pliable and elastic, so it can easily be stretched and molded directly on the patient's head after being rinsed to remove hot water droplets. After about 3 minutes the mask hardened and was ready for use. Patient details such as name, RT number, head rest used and date of fabrication of thermoplastic mask were written on it.

The patient was then taken for simulation and treatment planning, where by a conventional Tera-Six simulator was used to plan the treatment of a patient. The patient was positioned supine and aligned straight on the simulator table. An immobilization device was used to ensure reproducibility and rigidity of the setup. The area to be simulated was verified under fluoroscopic guidance. The isocenter placement was performed basing on physician instruction and field size of the area to be treated was determined with consideration of organs at risk. The beam placement design was performed by manipulating gantry and collimator angle to produce setup that would achieve minimum irradiation to normal tissues After beam design had been made, markup was done by using laser system to provide reference marks on patient skin or in the immobilization device, so that this position can be reproduced throughout the course of treatment. The final step in simulation process was documentation of patient setup data for calculation of treatment time figures 1,2 , show a photo of a thermoplastic mask used for immobilization of head and neck cancer at ORCI. 
LS-2000 is a rigid fixation frame designed to stabilize and restrict movement of the patient's head during treatment planning and delivery. It has fixation points that can clip alongside base plate which when combined with head rest gives optimal patient position for treatment planning and delivery. There are two adjustable knobs, one on either side that can be secured to labeled points on frame depending on the location of the disease in head and neck area, and comfortability of patient. During simulation, the patient was aligned in a straight comfortable position by using saggital laser. The patient laid head in a best fit head rest placed on the base plate. LS-2000 frame was fastened alongside base plate in a specified labeled fixation points. These labeled fixation points were well documented after simulation had been finished, so that they can be used to reproduce the plan during treatment delivery.

Verification images were taken on days 1-3 of treatment. The patient was laid in a supine position. The room lasers were used to align the patient in such a way that the saggital laser bisected the nasal septum, sternal notch, xiphisternum, and symphysis pubis as much as possible to ensure straightness and minimized rotation. Treatment isocenter was placed according to setup reference parameters. Radiotherapy field image was acquired by the use of verification film put into the special cassette. The cassette was placed perpendicular to the beam exiting the patient by the help of cassette stand. The film was exposed for short treatment time usually about 0.05 minutes for head and neck cases. The cassette was then removed after it had been exposed and taken to the dark room for the film to be processed by the automatic film processor figure 3 .

\section{Imaging}

Right or left lateral portal images were captured using a verification film. When taking portal image, a lead made cross wire was put on the center of the treatment field drawn on the thermoplastic mask during simulation, to mark the center of the treatment field on the portal image for thermoplastic mask immobilized group of patients. For the group of patients immobilized with an LS-2000 device, the lead made cross wire was put on the patient's skin at the center of treatment field which was obtained using reference of the treatment field center to prominent surface landmark of head and neck, mostly tragus when simulation was done. The portal images were obtained and processed in the automatic film processor in dark room. The images were produced and imported onto a viewing box illuminator.

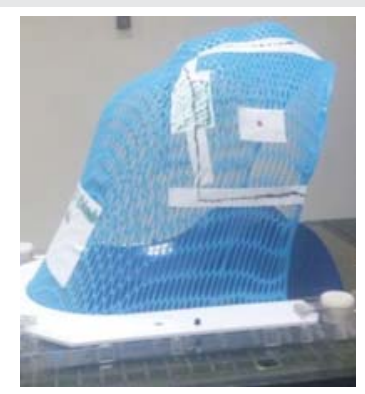

Figure 1: Thermoplastic mask and its accessories.

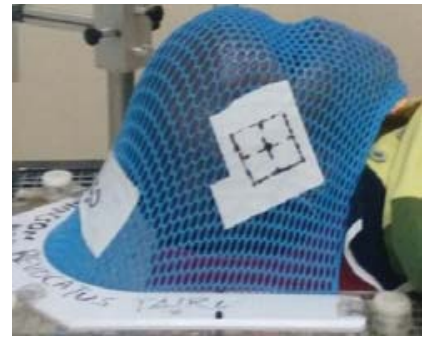

Figure 2: Supine positioned patient, with thermoplastic mask immobilizing device set up on a CO-60

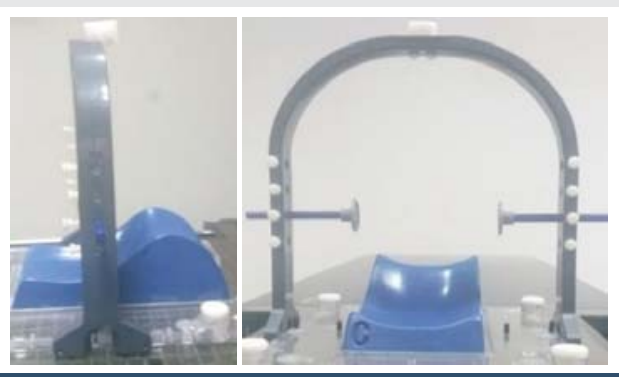

Figure 3: LS-2000 immobilization device and its accessories.

\section{Image analysis}

Corresponding simulator images were printed to obtain a hard copy to be used for comparison with portal images. With the help of two experienced radiotherapists, bony landmarks were identified on both portal images and respective simulator images, and structures to be matched were marked. For head and neck region, the treatment center may be referenced to various bony landmarks such, as supraorbital margin (SOM), angle of mandible, tip of mandible base of skull and cranial border of maxilla. Evaluation of translation setup errors in $\mathrm{AP}$ and SI direction were done by measuring distance from the center of the treatment field (isocenter) to clearly visible bony landmarks. Measurement was done manually using a transparent $30 \mathrm{~cm}$ ruler. For the purpose of documentation and analysis anterior and superior shifts were coded as positive shifts, whileposterior and inferior as negative shifts. The reproducibility of treatment field placement is reflected by a combination of both systematic and random error [7]. The systematic error is the deviation between the simulated or planned patient position and the average patient position. This deviation occurs in the same direction and is of a similar magnitude for each fraction throughout the treatment course [8]. Systematic error may occur at localization, planning or treatment delivery. Systematic error that was likely to occur during treatment delivery was assessed in this study. This error was obtained as the mean of all displacements in either the AP or SI direction [9]. The random error is found by subtracting the systematic displacement from the total displacement for one fraction [10].

For the "thermoplastic mask or LS-2000" group, the systematic error was the spread of individual mean errors for the group of patients, and the random error for the group was the standard deviation of all individual random errors. The aspects evaluated during statistical analysis were range of set up deviation, systematic error and random error associated with the two devices. The results were presented in tables 1-3. 


\section{Results}

A total of 90 portal images were acquired from 30 patients, 42 portal images for thermoplastic mask group and 48 portal images for LS-2000 immobilized patient group. These portal images were assessed in antero-posterior (AP) and superoinferior (SI) direction. The results of translational setup variations are presented in tables and figures as following.

\section{Range of setup variation}

The range of setup errors in both $\mathrm{AP}$ and SI direction for both groups of patients are given in table 1 .

The highest range of translational setup variation of -0.47 to $2.23 \mathrm{~cm}$ was found in LS-2000 immobilized patient group in the SI direction.

\section{Individual systematic setup variation}

The individual systematic setup variations for both groups of patients were shown in figures 4,5 , in each direction

The highest individual systematic variation for thermoplastic mask and LS-2000 immobilized patient groups is found to be 0.67 and $1.42 \mathrm{~cm}$, and 1.07 and $2.23 \mathrm{~cm}$ in AP and SI direction respectively.

For both patient groups, the individual systematic setup variation of less than $\pm 0.4 \mathrm{~cm}$ in the AP and SI directions were analyzed and shown in figure $6.75 \%$ and $50 \%$ of LS-2000 immobilized patients and $78.6 \%$ and $57.1 \%$ of the thermoplastic mask immobilized patients had average setup variation of less than $\pm 0.4 \mathrm{~cm}$.

\section{Random setup errors}

For all patients in each group, the random setup error was calculated as the standard deviation of the setup from the mean in each specified direction. The results for both groups are shown in table 2.

The random setup errors for LS-2000 immobilized patient

Table 1: The overall range of setup variation for both patient groups in the AP and SI direction.

\begin{tabular}{|c|c|c|}
\hline Patient group & AP(cm) & SI(cm) \\
\hline Thermoplastic mask & -0.53 to 0.67 & -0.53 to 1.07 \\
\hline LS-2000 & -0.8 to 1.42 & -0.47 to 2.23 \\
\hline
\end{tabular}

Table 2: The random setup variation of both patient groups in each direction.

\begin{tabular}{|c|c|c|}
\hline Patient group & $\mathrm{AP}(\mathrm{cm})$ & $\mathrm{SI}(\mathrm{cm})$ \\
\hline Thermoplastic mask & \pm 0.34 & \pm 0.48 \\
\hline LS-2000 & \pm 0.62 & \pm 0.64 \\
\hline
\end{tabular}

Table 3: The systematic setup errors of patient groups in each direction.

\begin{tabular}{|c|c|c|}
\hline Patient group & AP(cm) & SI(cm) \\
\hline Thermoplastic mask & \pm 0.35 & \pm 0.5 \\
\hline LS-2000 & \pm 0.62 & \pm 0.64 \\
\hline
\end{tabular}

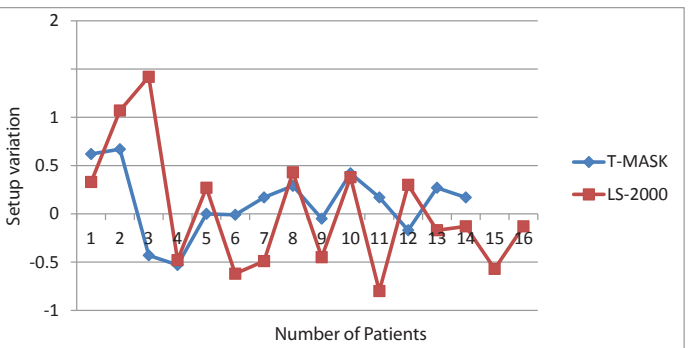

Figure 4: The distribution of individual patient systematic setup variation in AP direction.

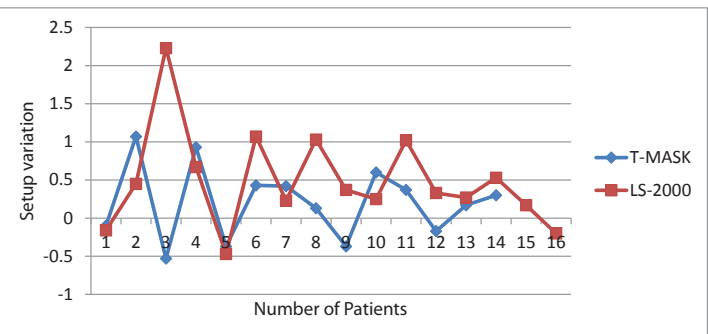

Figure 5: The distribution of individual patient systematic setup variation in SI direction.

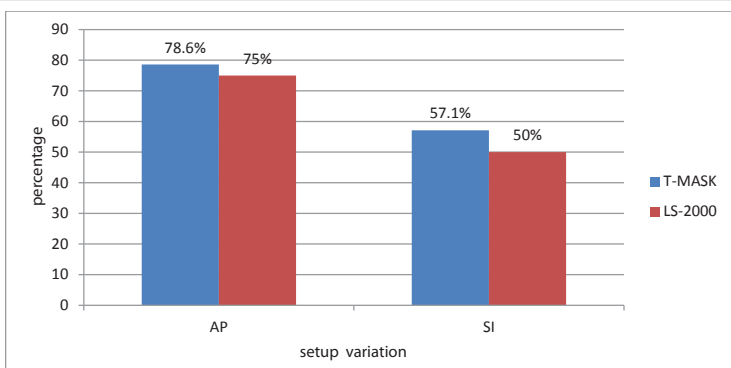

Figure 6: The percentage of patients with average setup variations of less than $\pm 0.4 \mathrm{~cm}$.

group were higher in both direction compared to thermoplastic mask immobilized group of patients.

\section{Systematic setup error}

The systematic setup error for all patients in each group was determined from the standard deviation of the values of the mean shifts (individual systematic setup variation) along a specified direction. Systematic setup variations are reduced for thermoplastic mask immobilized patient group in both directions. Results are shown in table 3, for both patient groups.

\section{Discussion}

Correct treatment field size coverage according to radiation treatment planning depends on the reproducibility of the patient position on a day-to-day basis throughout the course of treatment. In order to achieve treatment position reproducibility, HNC patients use immobilization devices for their treatment. This study attempts to evaluate comparatively the set-up accuracy and reproducibility of two immobilization devices used in our center in patients receiving conventional radiotherapy for head and neck cancers with lateral portals using verification films. The highest range of set up variation 
of $(-0.47$ to +2.23$) \mathrm{cm}$ was found in LS-2000 immobilized patient group in SI direction. This variation is reduced to $(-0.53$ to +1.07$) \mathrm{cm}$ for thermoplastic mask immobilized patient group in the same direction. This can be attributed to arbitrary motion of upper body part of patient after setup, since LS2000 does not completely restrict patient motion compared to thermoplastic masks. In AP direction, the highest range of -0.8 to 1.42 is found in LS-2000 immobilized group. This variation is reduced to -0.53 to +0.67 for thermoplastic immobilized patient group. This is also attributed to greater degree of freedom for patients immobilized with LS-2000. Generally the range of setup variation is greater in SI direction compared to AP direction.

These findings are slightly larger compared with the published head and neck paper using head and neck thermoplastic immobilization device [10]. reported the range of systematic errors of $[-2.5,2.7] \mathrm{mm}$ in the AP direction with a standard deviation of $1.9 \mathrm{~mm}$ and $[-3.6,2.8] \mathrm{mm}$ in the $\mathrm{C}-\mathrm{C}$ direction with a standard deviation of $1.8 \mathrm{~mm}$ in their study aimed to compare the positioning reproducibility and the cost efficiency for two head and neck immobilization devices. In this study they also assessed rotational errors. Gupta and his colleagues(2007) reported the mean displacement in AP; ML; and SI direction of $-0.25 \mathrm{~mm}$ (range -6.50 to $+7.70 \mathrm{~mm}$ ); -0.48 $\mathrm{mm}$ (range -5.50 to $+7.80 \mathrm{~mm}$ ); and $+0.45 \mathrm{~mm}$ (range -7.30 to $+7.40 \mathrm{~mm}$ ) respectively in their study which aimed to assess the set-up accuracy of head and neck RT using customized thermo- plastic immobilization andcompare with 'state-ofthe-art' practices [11].

This can be attributed partly to the limitation of the treatment delivery equipment and verification facilities used. Tanzania is among low and middle income countries where implementation of radiotherapy is faced by numerous challenges, including cost, infrastructure (electricity source and reliability) human resource and patient population. At the time of conducting this study, the center had only CO-60. One among of the limitation of $\mathrm{CO}-60$ is its dose distribution from its photon beams which are less conformal because of large source size and resulting larger penumbra (lateral falloff) for Co-60. This leads into fuzzy edge of the cobalt beam compared with the sharper field edge produced with linear accelerators used in the reported published findings.

Magnification factors during evaluation of portal images are associated with uncertainty. This uncertainty is caused by poor contrast resolution of bony landmarks used in image analysis. However, Emphasis was laid on the technique of manual measurements by precisely choosing the same points on reference and portal images. Random cross checking by a radiation oncologist ensured the quality of image analysis.

Seventy nine percent and seventy five percent of setup deviations werewithin $\pm 0.4 \mathrm{~cm}$ in AP direction for both thermoplastic mask and LS-2000 immobilization devices respectively. In SI direction fifty seven and fifty percent of setup deviation werewithin $\pm 0.4 \mathrm{~cm}$. In the AP direction, both groups of patients had better setup accuracy compared to the SI direction. This leads the investigator to question the comprehension of the set-up of the individual fields by the radiotherapists.

The number of thermoplastic mask immobilized patients within $\pm 0.4 \mathrm{~cm}$ average setup variation in both directions was larger compared to that in the LS-2000 immobilized group. This may be attributed to a better fit to the patient's anatomy since the thermoplastic mask can be fitted directly onto the patient.

Systematic errors and random errors were likely to be equal for the two immobilization devices in AP and SI direction, nevertheless larger errors are seen in LS-2000 immobilized patient group compared to thermoplastic mask group. This may be due to the fact that LS-2000 device does not completely restrict patient motion compared to the thermoplastic mask which normally extends beyond the treatment volume to provide necessary immobilization of the part to be treated.

\section{Conclusion}

This study quantified the accuracy and reproducibility of the head and neck immobilization devices used at ORCI. By comparing the deviations of both thermoplastic mask and LS2000 devices from isocenter as an indicator of the accuracy of patient repositioning, thermoplastic mask appeared to be an improvement over an LS-2000 device.

Knowing the limitation and setup variability of the device being used to set patients up for daily radiotherapy is paramount to planning and delivering appropriate radiation doses.

Increased accuracy of daily setup provides an opportunity to improve the therapeutic ratio both by increased likelihood of tumor control and decreased risk of normal tissue complications.

The two immobilization systems for HNC patients offered reasonable setup accuracy, in which the setup deviation was greater in SI direction than AP. This leaves the investigator to recommend that the radiotherapists need to be more vigilant when setting up head and neck cases, particularly in SI direction.

When comparing the two devices, the performance of the thermoplastic mask was better than LS-2000 as it demonstrated smaller translational deviations in both directions. The thermoplastic mask was recommended to be a superior device for use in immobilization of head and neck cancer treatment planning and delivery.

\section{References}

1. Yeh SA (2010) Radiotherapy for Head and Neck Cancer. Semin Plast Surg 24 127-136. Link: https://tinyurl.com/y8hns5wq

2. Mwansasu C, Liyombo E, Moshi N, Mpondo BCT (2015) Pattern of head and neck cancers among patients attending muhimbili national hospital tanzania. Tanzan J Health Res 17: 1-6. Link: https://tinyurl.com/y7rq9mvh

3. Gilyoma JM, Rambau PF, Masalu N, Kayange NM, Chalya PL (2015) Head and neck cancers: A clinico-pathological profile and management challenges in a resource-limited setting. BMC Res Notes 8: 1-9. Link: https://tinyurl.com/y9bkt36t 
4. Ratko T, Douglas GW, Souza J De, Belinson SE, Aronson N (2014) Radiotherapy Treatments for Head and Neck Cancer Update. Eff Heal Care Progr Comp Eff Rev 144. Link: https://tinyurl.com/ydzyjhpe

5. Cho HL, Park ET, Kim JKY, Kwak KS, Kim CJ, et al. (2013) Evaluation of radiotherapy setup accuracy for head and neck cancer using a 3-D surface imaging system. J Instrum 8: 11002-11002. Link: https://tinyurl.com/ydel9uyj

6. (2010) Comparative Effectiveness and Safety of Radiotherapy Treatments for Head and Neck Cancer - PubMed - NCBI. AHRQ Comp Eff Rev. Link: https://tinyurl.com/y8n3txqz

7. Belay EY (2011) (University of the witwatersrand). Evaluating setup accuracy of a positioning device for supine pelvic radiotherapy. Link: https://tinyurl.com/ya8tyh46

8. Howlin C, OShea E, Dunne M, Mullaney L, McGarry M, et al. (2018) A randomized controlled trial comparing customized versus standard headrests for head and neck radiotherapy immobilization in terms of set-up errors, patient comfort and staff satisfaction (ICORG 08-09). Radiography 21 74-83. Link: https://tinyurl.com/y9pt64v3

9. Das S, Isiah R, John S (2018) Comparison of setup errors based on bony landmarks in high precision radiotherapy in head and neck cancer: Results of a prospective study. Clin Cancer Investig J 4: 621. Link: https://tinyurl.com/ydchc399

10. Donato K, Leszczynski K, Fleming K (2006) A comparative evaluation of two head and neck immobilization devices using electronic portal imaging. $\mathrm{Br} J$ Radiol 79: 158-161. Link: https://tinyurl.com/ybeo3sn4

11. Gupta T, Chopra S, Kadam A, Agarwal J, Devi RP, et al. (2007) Assessment of three-dimensional set-up errors in conventional head and neck radiotherapy using electronic portal imaging device. Radiat Oncol 2: 1-8. Link: https://tinyurl.com/y7rq9mvh

\section{Highlights}

* Signatory publisher of ORCID

* Signatory Publisher of DORA (San Francisco Declaration on Research Assessment)

* Articles archived in worlds' renowned service providers such as Portico, CNKI, AGRIS, TDNet, Base (Bielefeld University Library), CrossRef, Scilit, J-Gate etc.

* Journals indexed in ICMJE, SHERPA/ROMEO, Google Scholar etc.

* OAI-PMH (Open Archives Initiative Protocol for Metadata Harvesting)

* Dedicated Editorial Board for every journal

* Accurate and rapid peer-review process

* Increased citations of published articles through promotions

* Reduced timeline for article publication

Submit your articles and experience a new surge in publication services (https://www.peertechz.com/submission).

Peertechz journals wishes everlasting success in your every endeavours.

Copyright: (c) 2018 Dukho AJ, et al. This is an open-access article distributed under the terms of the Creative Commons Attribution License, which permits unrestricted use, distribution, and $r$ eproduction in any medium, provided the original author and source are credited. 